Herzschr Elektrophys 2013 -24:67-68

DOI 10.1007/s00399-013-0255-9

Online publiziert: 9. April 2013

๑) Springer-Verlag Berlin Heidelberg 2013

\section{Hendrik Bonnemeier}

Abteilung für Elektrophysiologie und Rhythmologie, Klinik für Innere Medizin III,

Kardiologie, Angiologie, Intensivmedizin Universitätsklinikum Schleswig-Holstein, Kiel, Deutschland

\title{
Nichtinvasive Elektrophysiologie
}

Im Bereich nichtinvasive Elektrophysiologie gehörte im Jahr 2012 erneut die frühe Repolarisation („early repolarization“ - ER) in der internationalen Literatur zu den meist diskutiertesten Themen. Hier sind Daten hinsichtlich der Prävalenz einer ER bei herzgesunden Probanden sowie Prävalenz und Prognose einer ER in einer großen populationsbasierten Studie publiziert worden $[1,2]$. Ferner rückt mehr und mehr die prognostische Wertigkeit einer ER bei Patienten mit akuten Koronarsyndromen in den Fokus des Interesses [3, 4]. Hinsichtlich neuer nichtinvasiver elektrophysiologischer Parameter wurde erstmals ein interessanter Prädiktor für den plötzlichen Herztod beschrieben, der nach einer Belastungsuntersuchung die Erholung des räumlichen QRS/ T-Winkels beschreibt [5].

Eine frühe Repolarisation (ER) - definiert als eine J-Punkt-Elevation von $\geq 0,1 \mathrm{mV}$ in $\geq 2$ benachbarten Ableitungen - wird mit dem Auftreten von idiopathischem Kammerflimmern assoziiert. Über die Prävalenz einer ER bei herzgesunden Probanden gab es bislang keine ausreichenden Daten. Panicker et al. [1] haben digitalisierte Elektrokardiogramme von 1.886 herzgesunden Probanden, die an klinischen Phase-I-Studien teilgenommen haben, hinsichtlich ER untersucht. Mehr als ein Viertel (27,3\%) der Probanden wiesen im EKG eine ER auf. Hierbei zeigte der terminale Anteil des QRS-Komplexes eine Einkerbung („notching") bei $43 \%$ und einen trägen $\mathrm{Ab}$ stieg („slurring“) bei 57\% der Probanden mit ER. Die mittlere Herzfrequenz war bei Probanden mit ER signifikant niedriger. Rollin et al. [2] konnten ebenfalls eine hohe Prävalenz einer ER in einer populationsbasierten Studie an 1.161 südwestfranzösischen Einwohnern demonstrieren. Insgesamt 157 Einwohner (13,3\%) zwischen 35 und 64 Jahren zeigten eine ER. Während einer mittleren Nachverfolgung von 14 Jahren verstarben 77 Einwohner $(6,6 \%)$ - davon $24(2,1 \%)$ an kardiovaskulären Ursachen. Das Auftreten einer ER war mit signifikant erhöhten Risiken für Gesamtmortalität (Hazard-Ratio $2,45 ; p<0,001)$ und kardiovaskuläre Mortalität (Hazard-Ratio 5,6; $p<0,001$ ) assoziiert. Während eine ER mit einer Einkerbung („notching“) mit deutlich erhöhten Risiken assoziiert war (Gesamtmortalität Hazard-Ratio 3,84; kardiovaskuläre Mortalität Hazard-Ratio 8,75), war ein träger Abstieg („slurring“) des QRS-Komplexes nicht mit einer erhöhten Mortalität assoziiert. Somit liegt es nahe, dass zukünftig für die Risikostratifikation beim Vorliegen einer ER der „Notching-Pattern“ herangezogen werden sollte.

In zwei weiteren Studien [3, 4] wurde der Hypothese nachgegangen, dass das Vorliegen einer ER mit einer erhöhten Vulnerabilität für lebensbedrohliche Tachyarrhythmien während eines akuten koronaren Ereignisses/eines Myokardinfarkts einhergeht. In der Studie von Tikkanen et al. [3] wurden EKGs von 432 konsekutiven Patienten, welche an einem plötzlichen Herztod während eines akuten Koronarereignisses verstorben sind, mit EKGs von 532 Überlebenden eines akuten Koronarereignisses verglichen. Alle 12-Kanal-EKGs der Patienten wurden vor dem akuten Koronarereignis abgeleitet. Die Prävalenz einer ER war signifikant höher in der Gruppe der am plötzlichen Herztod verstorbenen Patienten (14,4\%) als in der Kontrollgruppe (7,9\%). Nach Adjustierung für Unterschiede in den Basisparametern zeigte sich für den plötzlichen Herztod während eines akuten Koronarereignisses eine Odds-Ratio von 2,15 bei Vorliegen einer ER. Während in der Studie von Tikkanen et al. EKGs unter- sucht worden sind, welche weit vor dem akuten Koronarereignis und ohne Bezug auf das Ereignis geschrieben wurden, wählten Rudic et al. [4] einen anderen Ansatz: Bei 60 Patienten mit akutem Myokardinfarkt (30 Patienten nach Reanimation bei Kammerflimmern während des Myokardinfarkts, 30 Patienten mit unkompliziertem Myokardinfarkt) wurden aktuelle EKGs analysiert. Bei insgesamt 18 Patienten (30\%) aller Patienten konnte eine ER gefunden werden, von denen vor allem (78\%) das „Notching-Pattern“ evident war. Patienten mit Kammerflimmern wiesen signifikant häufiger eine ER auf (47 vs. $13 \%$ ). Somit scheint das Vorliegen einer ER bei Eintritt eines akuten Koronarereignisses mit einer erhöhten Vulnerabilität für ventrikuläre Tachyarrhythmien assoziiert zu sein.

Ein neuartiger Prädiktor für den plötzlichen Herztod wurde in einer Studie an 1.297 Patienten, die einer klinisch indizierten Fahrradergometrie unterzogen wurden, getestet [5]. Es handelt sich um einen dynamischen Parameter, der den räumlichen Zusammenhang der Depolarisations- und Repolarisationswellenfronten („total consine T-to-R“ [TCRT]) in den ersten 3 min der Erholungsphase nach Belastung aus 12-Kanal-Elektrokardiogrammen beschreibt. Hieraus wurde die herzfrequenzkorrigierte TCRT/Herzfrequenz-Hysterese bestimmt. Während einer Nachverfolgung von 45 Monaten verstarben 2,6\% der Patienten an einem kardialen Tod und 1,9\% der Patienten an einem plötzlichen Herztod. Eine reduzierte TCRT/Herzfrequenz-Hysterese war hochsignifikant mit der kardialen Mortalität assoziiert. Auch nach Adjustierung für Unterschiede in den klinischen Basisparametern blieb der neue Parameter ein unabhängiger Prädiktor für den kardialen Tod (Hazard-Ratio 5,6). Da die Fahrrad- 
ergometeruntersuchung in der klinischen Praxis eine sehr häufige Routineuntersuchung darstellt und in der Regel mit computergestützten Systemen durchgeführt wird, könnte ein Upgrade mit einer kommerziellen Software zur automatischen Berechnung der TCRT/HerzfrequenzHysterese die Risikostratifikation kardiologischer Patienten zukünftig vereinfachen und einer breiteren Patientenpopulation zugänglich machen.

\section{Korrespondenzadresse}

\section{Prof. Dr. H. Bonnemeier}

Abteilung für Elektrophysiologie und Rhythmologie, Klinik für Innere Medizin III, Kardiologie, Angiologie

Intensivmedizin Universitätsklinikum

Schleswig-Holstein, Campus Kiel

Schittenhelmstr. 12, 24105 Kiel

hendrik.bonnemeier@uk-sh.de

Interessenkonflikt. Der korrespondierende Autor gibt an, dass kein Interessenkonflikt besteht.

\section{Literatur}

1. Panicker GF, Manohar D, Karnad DR, Salvi V, Kothari S, Lokhandwala Y (2012) Early repolarization and short QT interval in healthy subjects. Heart Rhythm 9:1265-1271

2. Rollin A, Maury P, Bongard V, Sacher F, Delay M, Duparc A, Mondoly P, Carrié D, Ferrières J, Ruidavets JB (2012) Prevalence, prognosis, and identification of the malignant form of early repolarization pattern in a population-based study. Am J Cardiol 110:1302-1308

3. Tikkanen JT, Wichmann V, Junttila MJ, Rainio M, Hookana E, Lappi OP, Kortelainen ML, Anttonen O, Huikuri HV (2012) Association of early repolarization and sudden cardiac death during an acute coronary event. Circ Arrhythm Electrophysiol 5:714718

4. Rudic B, Veltmann C, Kuntz E, Behnes M, Elmas E, Konrad T, Kuschyk J, Weiss C, Borggrefe M, Schimpf $R$ (2012) Early repolarization pattern is associated with ventricular fibrillation in patients with acute myocardial infarction. Heart Rhythm 9:1295-1300

5. Kentää T, Viik J, Karsikas M, Seppänen T, Nieminen T, Lehtimäki T, Nikus K, Lehtinen R, Kähönen M, Huikuri HV (2012) Postexercise recovery of the spatial QRS/T angle as a predictor of sudden cardiac death. Heart Rhythm 9:1083-1089 